\title{
Small Molecules Targeting the Inactive Form of the Mnk1/2 Kinases
}

Srinivasaraghavan Kannan, ${ }^{*},{ }^{\dagger}$ Mohan R. Pradhan, ${ }^{\dagger}$ Joseph Cherian, $^{\ddagger}{ }^{\dagger}$ Thomas L. Joseph, ${ }^{\dagger}$ Zhi Ying Poh, ${ }^{\ddagger}$ Yang Hai Yan, ${ }^{\ddagger}$ Ho Melvyn, ${ }^{\ddagger}$ Liu Boping, Hill Jeffrey, ${ }^{\ddagger}$ Kassoum Nacro, and Chandra S. Verma*,†, ${ }^{*}, \|$

${ }^{\dagger}$ Bioinformatics Institute (A*STAR), 30 Biopolis Street, \#07-01 Matrix, Singapore 138671, Singapore

${ }^{\ddagger}$ Experimental Therapeutics Centre (A*STAR), 31 Biopolis Street, \#03-01 Nanos, 138669, Singapore

${ }^{\S}$ School of Biological Sciences, Nanyang Technological University, 60 Nanyang Drive, 637551, Singapore

"Department of Biological Sciences, National University of Singapore, 14 Science Drive 4, 117543, Singapore

Supporting Information
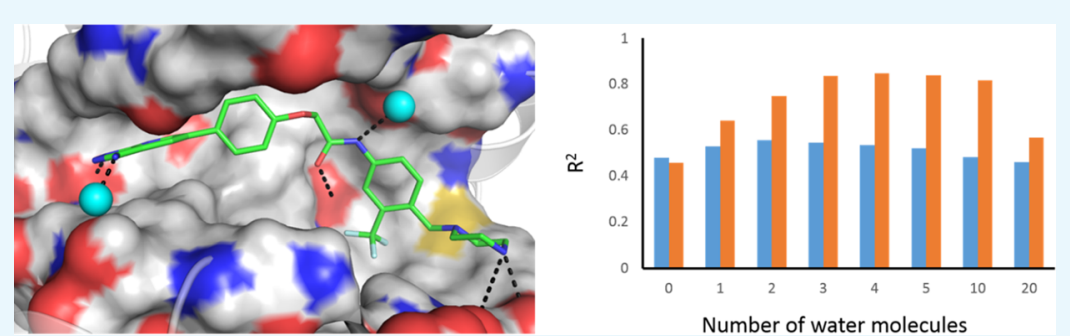

ABSTRACT: Overexpression of the eukaryotic initiation factor 4E (eIF4E) is linked to a variety of cancers. Both mitogenactivated protein kinases-interacting kinases 1 and $2(\mathrm{Mnk} 1 / 2)$ activate the oncogene eIF4E through posttranslational modification (phosphorylating it at the conserved Ser209). Inhibition of Mnk prevents eIF4E phosphorylation, making the Mnk-eIF4E axis a potential therapeutic target for oncology. Recently, the design and synthesis of a series of novel potent compounds inhibiting the Mnk1/2 kinases were carried out in-house. Here, we describe computational models of the interactions between Mnk1/2 kinases and these inhibitors. Molecular modeling combined with free energy calculations show that these compounds bind to the inactive forms of the kinases. All compounds adopt similar conformations in the catalytic sites of both kinases, stabilized by hydrogen bonds with the hinge regions and with the catalytic Lys78 (Mnk1) and Lys113 (Mnk2). These hydrogen bond interactions clearly play a critical role in determining the conformational stability and potency of the compounds. We also find that van der Waals interactions with an allosteric pocket are key to their binding and potency. Two distinct hydration sites that appear to further stabilize the ligand binding/interactions were observed. Critically, the inclusion of explicit water molecules in the calculations results in improving the agreement between calculated and experimental binding free energies.

\section{INTRODUCTION}

Mitogen-activated protein kinases-interacting kinases 1 and 2 $(\mathrm{Mnk} 1 / 2)$ are Ser/Thr kinases from the $\mathrm{Ca}^{2+} /$ calmodulindependent kinase family. They both activate the eukaryotic initiation factor $4 \mathrm{E}$ (eIF4E) by phosphorylating it at the conserved Ser209. ${ }^{1-3}$ eIF4E initiates the translation of messenger RNAs into proteins. It has been found to be overexpressed in several cancers. ${ }^{4}$ This makes the Mnk-eIF4E axis a potential therapeutic target for oncology. ${ }^{5-7}$ Indeed, it has been demonstrated that simultaneous inhibition of an oncogenic kinase (mTOR, BCR-ABL, and MEK) together with that of Mnk kinases results in downregulation of protein synthesis, cell cycle progression, and proliferation of prostate cancer cells and chronic myeloid leukemia cells in the blast crisis stage, and in neurofibromin 1 mutant cancers. ${ }^{8-10}$ There is a growing interest in developing Mnk inhibitors even as two compounds have recently entered clinical trials. ${ }^{1,12}$

The catalytic domains of Mnk1/2 share $\sim 80 \%$ sequence identity. In contrast to other protein kinases, Mnk1/2 kinases contain special inserts (I1, I2, and I3) and a distinct Asp-PheAsp (DFD) motif (Figure S1). The experimental structures of the active conformations of the kinase domains of Mnk1 and Mnk2 display a bilobal catalytic domain seen in protein kinases, which sandwiches the adenosine 5'-triphosphate (ATP) pocket between the $\mathrm{N}$ - and $\mathrm{C}$-terminal lobes (Figure $\mathrm{S} 1$ ). The $\mathrm{N}$ terminal lobe is made up of a twisted sheet of five antiparallel $\beta$ strands $(\beta 1-\beta 5)$ and the regulatory helix $\alpha \mathrm{C}$. It harbors the $\mathrm{P}$ loop (residues 51-62 in Mnk1 and 86-97 in Mnk2) which is rich in glycines, a conserved lysine (Lys78 in Mnk1 and Lys113 in Mnk2), and a conserved glutamic acid (Glu94 in Mnk1 and Glu129 in Mnk2) (Figure 1). These two residues form a critical salt bridge which is conserved across the active conformations of kinases. Together, these elements orchestrate the binding of ATP. The C-terminal lobe is largely made up on $\alpha$-helices and

Received: September 20, 2017

Accepted: October 31, 2017

Published: November 14, 2017 


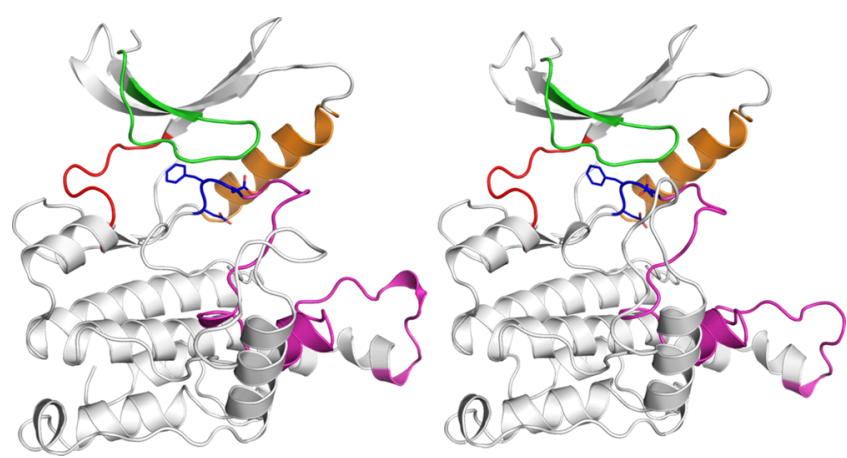

Figure 1. Models of Mnk1 and Mnk2 kinases based on homology. Different structural elements are highlighted: P-loop (green), hinge region (red), catalytic loop (magenta), DFD motif (blue), and $\alpha \mathrm{C}$ helix (orange).

binds the peptide substrate and enables phosphate transfer. The Mnk kinases are characterized by a noncanonical As$\mathrm{p}^{191} \mathrm{Phe}^{192} \mathrm{Asp}^{193}$ motif in Mnk1 and $\mathrm{Asp}^{226} \mathrm{Phe}^{227} \mathrm{Asp}^{228}$ motif in Mnk2; this contrasts with the more common Asp-Phe-Gly motif.

Mnk1 has been crystallized in an autoinhibited form (PDB id 2HW6, resolution $2.6 \AA) .{ }^{13}$ Phe230 from the Mnk1-specific insertion region lies in a hydrophobic allosteric pocket which lies adjacent to the ATP binding pocket; the pocket is created by the residues Glu94, Leu98, Cys101, Gln102, Ile107, Leu108, Leu161, Ile166, Ala167, His168, Ile189, and Cys190 in Mnk1 (Figure S2). In other kinases, the active state is characterized by the Phe from the DFG motif inserted into this hydrophobic allosteric pocket, whereas in the inactive state, the Type II inhibitors occupy this region. A crystal structure of the Mnk2 kinase in a DFD-out state has also been resolved (PDB id $2 \mathrm{AC} 3$, resolution $2.1 \AA) .{ }^{14}$ In contrast to Mnk1, Mnk2 is not autoinhibited, and the activation loop adopts an extended conformation. Similar to other kinases, in the inactive form of the Mnk2 kinase, the displacement of Phe158 from the DFD motif (its DFG motif in other kinases) creates an allosteric pocket adjacent to the ATP binding pocket constituted by residues Glu129, Leu133, Cys136, Gln137, Val142, Leu143, Leu196, Ile201, Ala202, His203, Ile223, and Cys224. Structural information for a majority of the activation loop (A-loop) and Mnk-specific insertions is missing.

The most investigated group of Mnk kinase inhibitors are ATP-competitive molecules that bind to the active conformation (DFD-in) of the kinases, mimicking the interactions of the adenine ring of ATP; these are referred to as the Type I inhibitors, as has been highlighted earlier too. ${ }^{15}$ Recently, a novel series of inhibitors that target the inactive conformations of the Mnk kinases with nanomolar to micromolar affinities have been developed in-house; ${ }^{16-18}$ these are referred to as the Type II inhibitors. We report here a study that combines molecular modeling and molecular dynamics (MD) simulations exploring the kinase-inhibitor interactions. We had earlier used a similar protocol to successfully probe the binding of novel Type I (imidazopyridine and imidazopyrazine derivatives) Mnk1/2 inhibitors. ${ }^{15}$ In this study, we further report that water molecules play a novel role by stabilizing the interactions of the Type II inhibitors with the Mnk1/2 kinases.

\section{RESULTS AND DISCUSSIONS}

Homology Modeling and Refinement. There are no atomic structures of wildtype Mnk1/2 in their inactive states; hence, we used the available crystal structure of the calmodulindomain protein kinase $1^{19}$ ( $\sim 35 \%$ amino acid identity) in its inactive state as a template to build their models; these models were additionally constrained by the available structures of Mnk1/2 as mentioned in the Materials and Methods section. The generated models are structurally similar to the templates used, with a root mean squared deviation ( $\mathrm{rmsd}$ ) ranging from $\sim 1.3$ to $1.5 \AA$. Unlike the crystal structure of the Mnk1 kinase, the modeled structure is in the inactive form of the kinase, with both the ATP binding pocket and the adjacent allosteric pocket accessible to inhibitors. These models were refined by atomistic $\mathrm{MD}$ simulations in explicit water. During the MD simulations, both kinases remained stable (within $\sim 3-4 \AA$ of the non Aloop regions of the starting states); only the A-loop exhibited large flexibility and conformational changes (Figure 2).

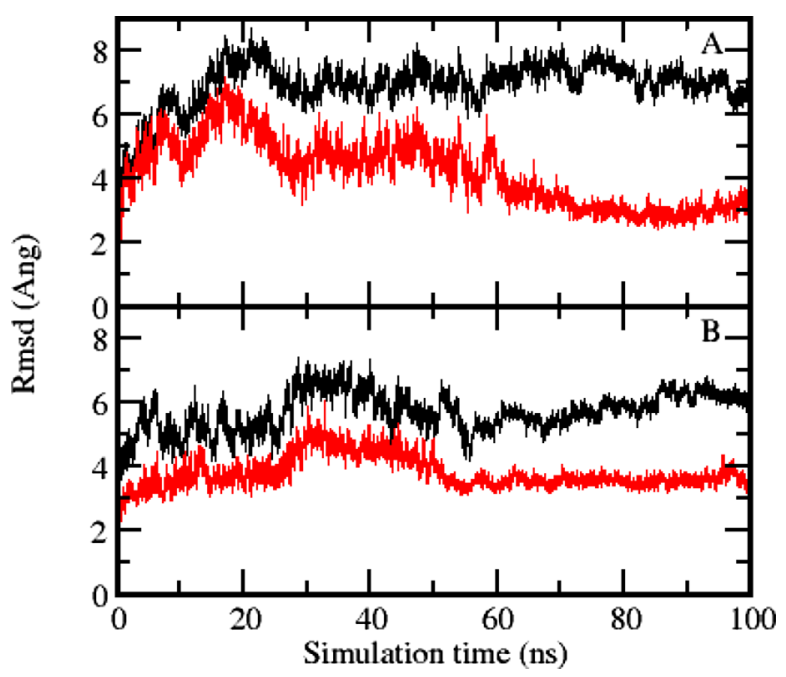

Figure 2. Structural deviations of the modeled conformations of Mnk1 (A) and Mnk2 (B) sampled during the MD simulations. RMSD (root mean square devialtion) is calculated after superposition of each conformation from the MD trajectory on the respective homology models of all residues (black), excluding the activation loop (red).

The ligand binding pocket in the $M n k 1 / 2$ kinases share $\sim 85 \%$ sequence identity with very similar overall electrostatic potentials and small differences in the hinge region, P-loop, and at the allosteric pocket (Figure 3).

Ligand Docking. A set of Type II small-molecule inhibitors that were developed in-house ${ }^{17}$ and have been shown to inhibit the activity of Mnk kinases with $\mathrm{IC}_{50}$ values ranging from $\mathrm{nM}$ to $\mu \mathrm{M}$ (Table 1 ) were docked into the catalytic sites of $\mathrm{Mnk} 1 / 2$

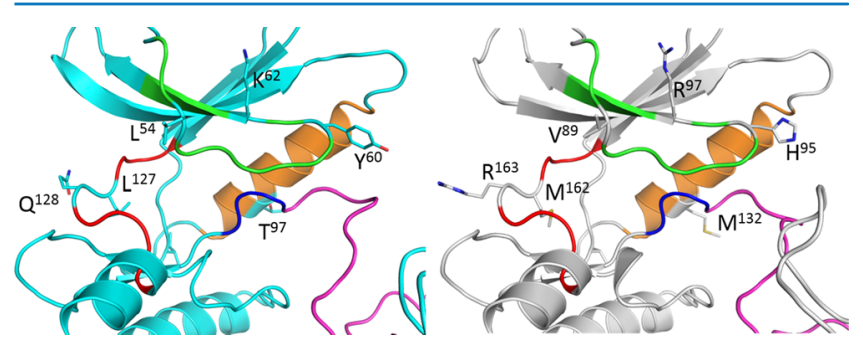

Figure 3. Differing residues in the active sites of Mnk1 (left) and Mnk2 (right) are highlighted as sticks and labeled accordingly; structural elements are highlighted: P-loop (green), hinge region (red), catalytic loop (magenta), DFD motif (blue), and $\alpha \mathrm{C}$ helix (orange). 
ACS Omega

Article

Table 1. Structure and Activities of Type II Ink Inhibitors ${ }^{a}$

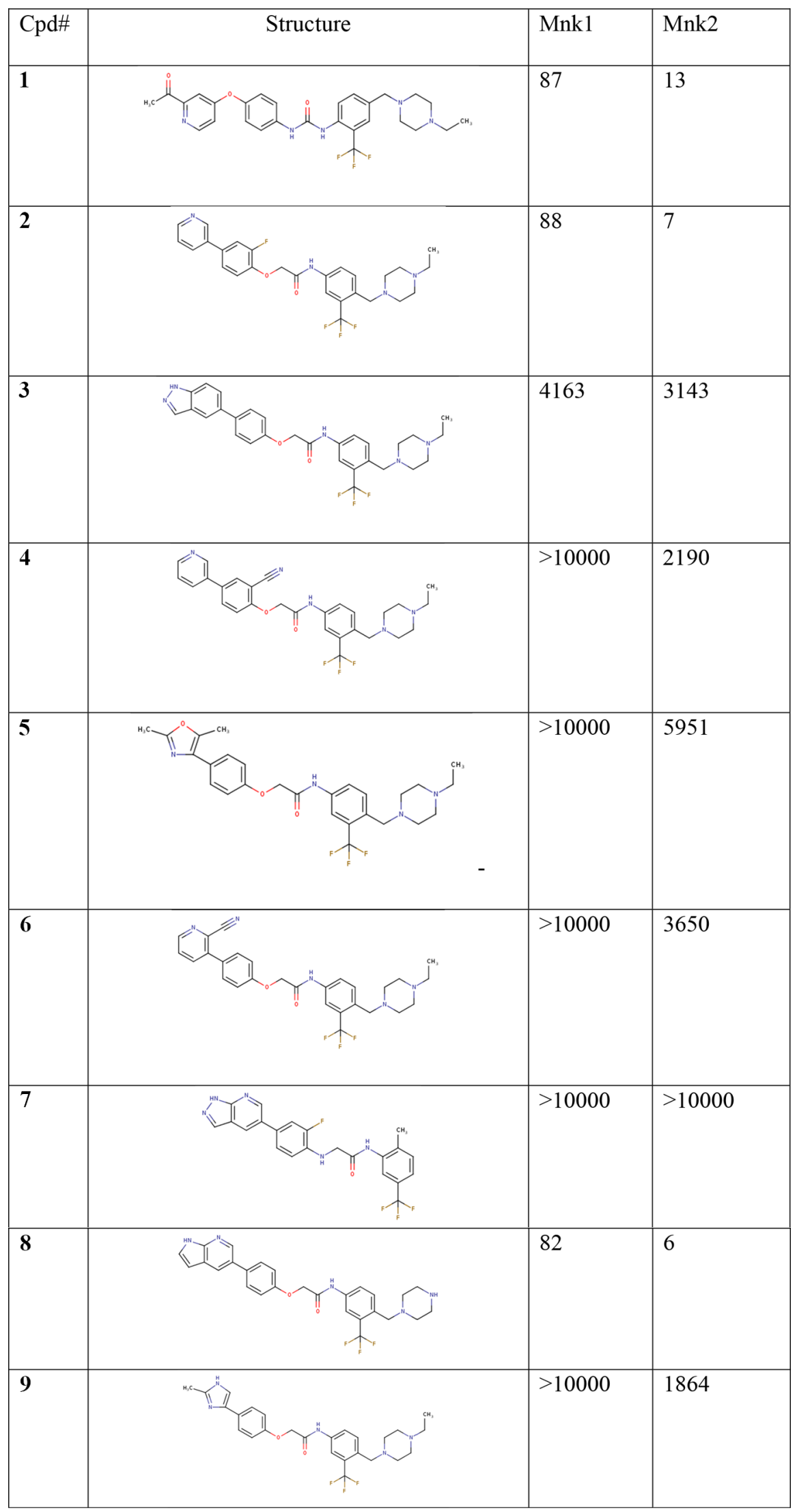

7883

DOl: $10.1021 /$ acsomega.7b01403

ACS Omega 2017, 2, 7881-7891 
ACS Omega

Article

Table 1. continued

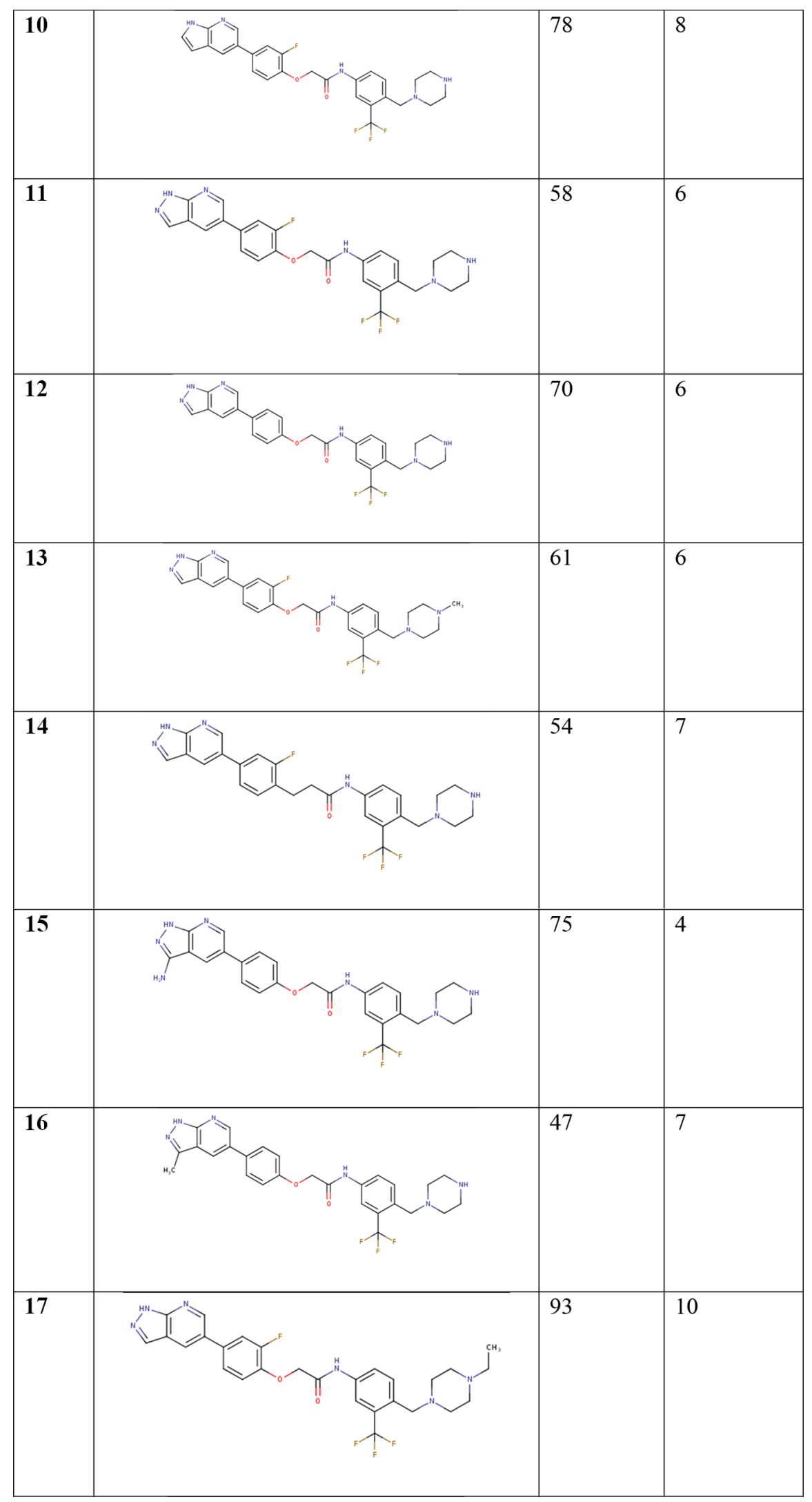

7884

DOI: 10.1021/acsomega.7b01403

ACS Omega 2017, 2, 7881-7891 
Table 1. continued

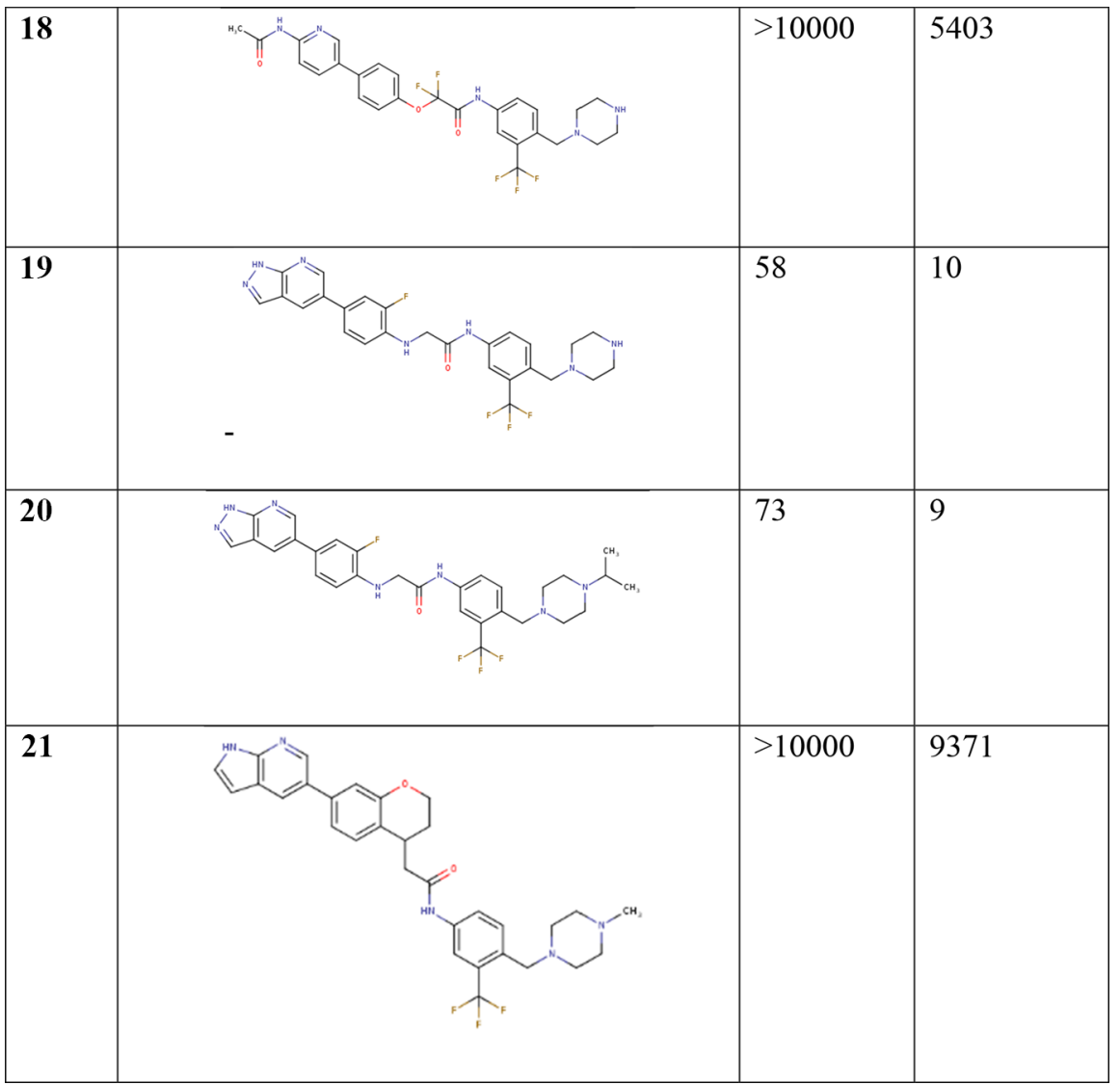

${ }^{a}$ Activities are given as inhibition constant $\left(\mathrm{IC}_{50}\right)$; values are in nanomolar $(\mathrm{nM})$.

kinases in their modeled active (DFD-in) and inactive (DFDout) conformations. For docking calculations, a protocol that was developed and benchmarked earlier ${ }^{15}$ to successfully probe the binding of novel (imidazopyridine and imidazopyrazine derivatives) Mnk1/2 inhibitors was used. Reasonable docked poses were observed only for the inactive forms of the kinases. As these compounds are relatively large, binding of these compounds requires a large pocket which exists only in the inactive form of the kinase (Figure S3; in the active form, the restricted pocket size results in the docked conformations with the compound largely exposed to the solvent). The compounds considered in this study are also structurally similar to other kinase inhibitors that are known to bind to the inactive forms of the kinases, such as imatinib bound to BCR-ABL (PDB 2HYY) and ponatinib bound to BCR-ABL (PDB 3OXZ), for which several crystal structures are available. On the basis of this, we chose the compounds bound to the inactive forms of Mnk1/2 for subsequent analysis. On the basis of the binding mode, parts of these compounds can be referred to as the following: (1) hinge binders: parts of compounds that interact with the hinge region of the kinase, (2) allosteric pocket binders: parts of compounds that occupy the allosteric pocket created by the flipping of Phe in the DFD-out conformation, (3) linker region: parts of compounds that connect the hinge binders with the allosteric pocket binders, and (4) tail part: the extension of the allosteric binders and are regions that interact with the A-loop (Figure 4).

Examining the models of the compounds listed in Table 1 docked to Mnk1/2, it can be seen that the ATP pocket is occupied by pyridine (compounds 2 and 4 ), cyanopyridine

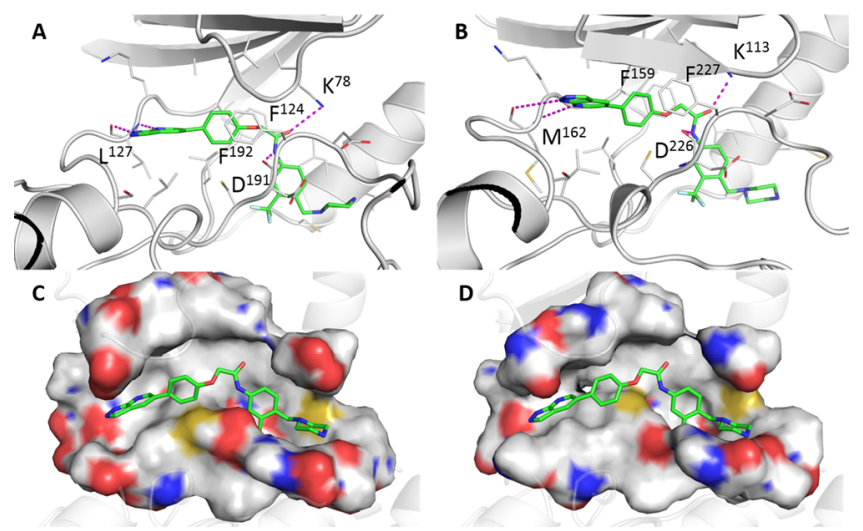

Figure 4. Predicted binding mode of compound 11 to Mnk1 (A,C) and Mnk2 (B,D). (Top) Active site residues are shown as lines; hydrogen bonds are indicated by dashed lines (magenta); kinase residues involved in hydrogen bond interactions are labeled. (Bottom) Compound $\mathbf{1 1}$ is shown bound to the active site in surface.

(compound 6), acetopyridine (compound 1), acetamidopyridine (compound 18), indazole (compound 3), pyrrolo(3,4b)pyridine (compounds 8, 10, and 21), pyrazolopyridine (compounds $7,11,12,13,14,15,16,17,19$, and 20), dimethylfuran (compound 5), and methylimidazole (compound 9). The substituents on these 5- or 6-membered rings interact with the hinge region through one or two hydrogen bonds with the backbone atoms of Leu127 (Mnk1) or Met162 (Mnk2) and through van der Waals (vdW) packing interactions with residues from the hinge region and the P-loop. It is clear 

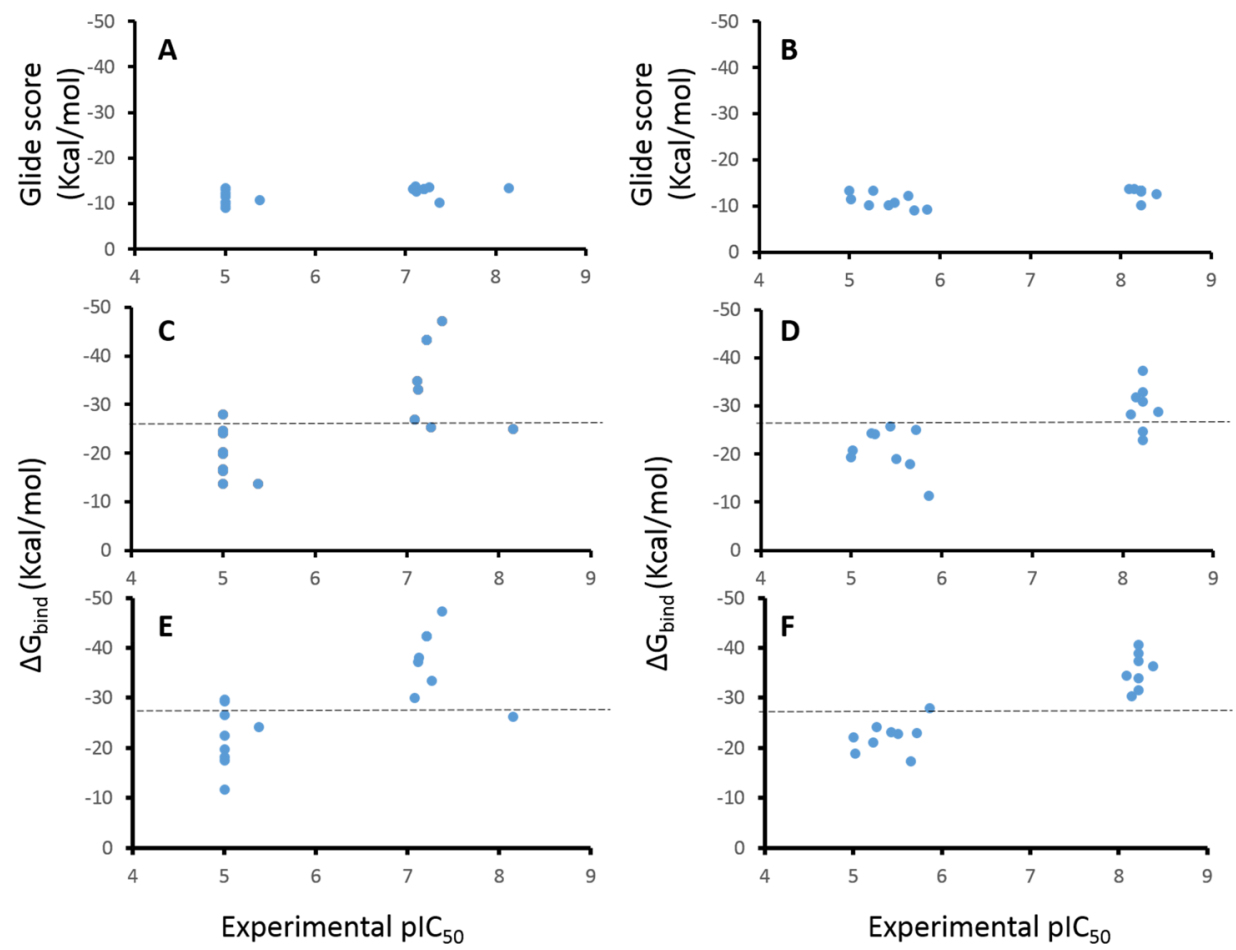

Figure 5. Correlation between the calculated $(\mathrm{A}, \mathrm{B})$ dock score (glide energy) and MMPBSA binding free energies $\left(\Delta G_{\text {bind }}\right)$ without water $(\mathrm{C}, \mathrm{D})$ and with water (E,F) and the experimental $\mathrm{pIC}_{50}$ values for (left) Mnk1 and (right) Mnk2. The dashed lines indicate the separation of binders and binders based on the predicted binding energies.

that the hydrogen bonds in the ATP binding pocket are key determinants of affinity because their removal (compounds 3, 5, and 9) results in reduced potency. In addition, the trifluoromethyl moiety, common to all molecules, occupies the allosteric pocket formed by residues Cys101, Ile59, Leu113, Ile141, and Cys142 in Mnk1 and Cys136, Val142, Leu196, Ile224, and Cys225 in Mnk2. These interactions are all driven by vdW interactions. The allosteric binder is another important contributor for the binding and potency of Type II inhibitors, as most of this class of inhibitors has either a phenyl or a halogenated phenyl as the allosteric binder. The linker regions are made up of phenoxyacetamide (compounds 3, 4, 5, 6, 8, 9, 12,15 , and 16), flurophenoxyacetamide (compounds 2, 10, 11, 13, and 17), flurobenzylacetamide (compound 14), hydroxyphenylurea (compound 1), fluroanilinoacetamide (compound 7, 19, and 20), dihydrobenzoxazinacetamide (compound 21), and difluoro-phenoxyacetamide (compound 18). The linker amide (except compound 1) is involved in hydrogen bond interactions with the backbone carbonyl of Asp191 (Mnk1)/ Asp226 (Mnk2) and with the side chain of catalytic Lys78 (Mnk1)/Lys113 (Mnk2). Compound 1 has a urea as the linker but is involved in hydrogen bond interactions which are similar to those made by amide linkers. Although most linkers are welltolerated, substitutions such as difluoro as in the case of compound $\mathbf{1 8}$ are clearly not tolerated; this results from the substitutions sterically clashing with the kinases at this position. The phenoxy group from the linkers is sandwiched between Phe124 (gate keeper residue) and Phe219 from the DFD motif in Mnk1 and Phe158 (gate keeper residue) and Phe227 from the DFD motif in Mnk2. Both the unsubstituted (compounds $1,3,5,6,8,9,12,15,16$, and 18) and fluoro-substituted phenoxy-containing compounds (compounds 2, 7, 10, 11, 13, $14,17,19$, and 20) have comparable activity; however, other substitutions (cyanophenoxy in compound $\mathbf{4}$ and dihydroben- zoxazin in compound 21) are not tolerated as the large substitutions will have steric clashes at this position and resulted in reduced potency with $\mathrm{IC}_{50}$ in the micromolar range. The tail regions of all compounds contain either piperazine (compounds 8, 10, 11, 12 14, 15, 16, 18, and 19) or substituted piperazine, that is, methylpiperazine (compounds 13 and 21), ethylpiperazine (compounds 1, 2, 3, 4, 5, 6, 9, and 17), or isopropylpiperzine (compound 20). The nitrogen atom from the piperazine engages in hydrogen bond interactions with the carbonyl backbone of Ala167 (Mnk1)/Ala202 (Mnk2) and is the most diverse region among the compounds. It is clear that the tail region interaction is also very important for activity, as compound 7 which lacks the tail region has reduced potency.

The resultant docking scores showed a poor correlation with experimental data (Figure 5A,B); this is not unexpected from current docking approaches. ${ }^{20}$ To circumvent this, we subjected all docked complexes to MD simulations.

MD Simulations of Protein-Inhibitor Complexes. The stability of the complexes during the MD simulations as judged by their structural deviations was deemed satisfactory (rmsd of $\sim 4 \AA$ in the receptor atoms excluding the activation loop; Figure 6). The bound Type II inhibitors do not deviate by more than $\sim 2 \AA$ from their starting conformations. Although we did not witness the exit from the binding pocket of any of the inactive compounds, they did show increased flexibility when bound to Mnk1 (Figure 6). While it is known that unbinding events probably require simulations of much longer timescales, ${ }^{21}$ in our study, extension of MD simulations to a microsecond did not show any unbinding event.

The protein-inhibitor interactions were generally conserved during the MD simulations. Hydrogen bonds between the inhibitors and receptors, especially the interactions with the hinge regions of kinases, are known to play a key role in drug inhibition. ${ }^{22}$ Several hydrogen bonds between the inhibitors 


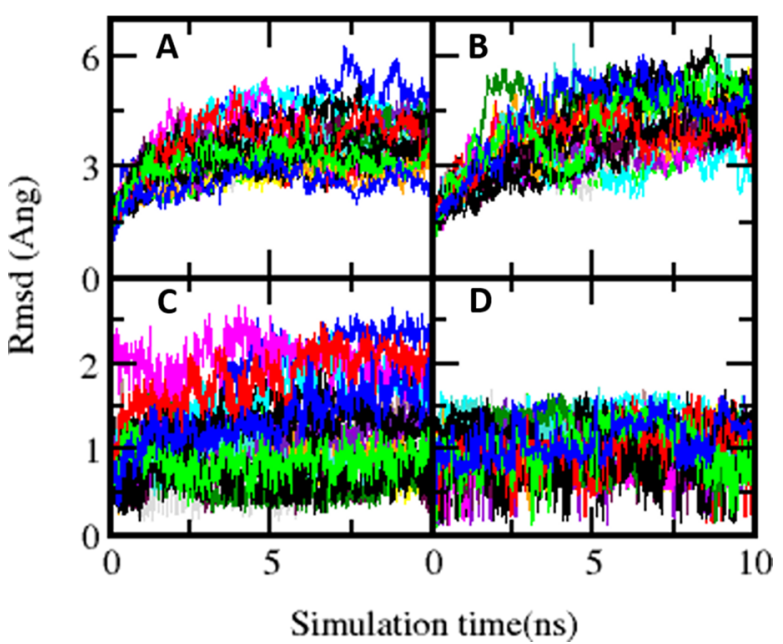

Figure 6. Structural deviations from the docked models of the Mnkinhibitor complexes sampled during the MD simulations. RMSD of Mnk1 (A) and Mnk2 (B); rms of compounds bound to Mnk1 (C) and Mnk2 (D).

and the kinases are seen in the simulations. The hydrogen bonds between the molecules and the Leu127-NH/Met162$\mathrm{NH}$ located in the hinge regions of Mnk1/2 were stable (except compounds 5 and 9) during the simulations. The fact that the two weak inhibitors, compounds 5 and $\mathbf{9}$, do not form this hydrogen bond, suggests that this interaction is important. It is likely that the absence of this hydrogen bonding results in increased flexibility of compounds 5 and 9 during the simulations. Two different kinds of interactions were observed for the amide linker: (1) hydrogen bonding between the Asp191-NH/Asp226-NH in Mnk1/2 and linker oxygens, and between the side chain oxygens of Glu94/Glu129 in Mnk1/2 and the linker amide nitrogen and (2) hydrogen bonding between the backbone carbonyl of Asp191 in Mnk1/Asp226 in Mnk2 with linker amide nitrogen, and between the side chain nitrogen of Lys78 in Mnk1/Lys113 in Mnk2 with linker carbonyl oxygen. In addition to the hydrogen bond at the hinge region, the hydrogen bond involving the amide linker is important for stabilizing the bound conformations of these inhibitors. The stacking between the phenyl ring of the phenoxy group in all compounds with Phe124 and Phe219 in Mnk1 and Ph158 and Phe227 in Mnk2 is also well-preserved in those cases where the compound is involved in stable interactions with the hinge region. The trifluoromethyl $\left(\mathrm{CF}_{3}\right)$ moiety remains buried into the hydrophobic pocket and hydrogen bond interactions between the positively charged piperazine, and Ala167 (Mnk1)/Ala202 (Mnk2) are wellpreserved throughout the simulations.

In addition to the contacts made directly between the inhibitors with the kinase residues, water molecules are also known to play a critical role in inhibitor recognition, stabilization, and potency. ${ }^{23-27}$ The structured water molecule is also thought to modulate selectivity among kinase inhibitors. $^{24-29}$ In our simulations, we find two distinct hydration sites. The first site corresponds to the hinge region, where we found water molecules (different numbers of water molecules for different inhibitors) with high residence times. Some of these water molecules are also involved in watermediated hydrogen bonds between the kinases and the inhibitors. For example, amine nitrogen from the hinge binder of compound 16 is involved in water-mediated hydrogen bond interactions with the side chains of Ser131 and Ser166 from Mnk1 and Mnk2, respectively, and this interaction is wellpreserved throughout the simulations (Figure 7). The second

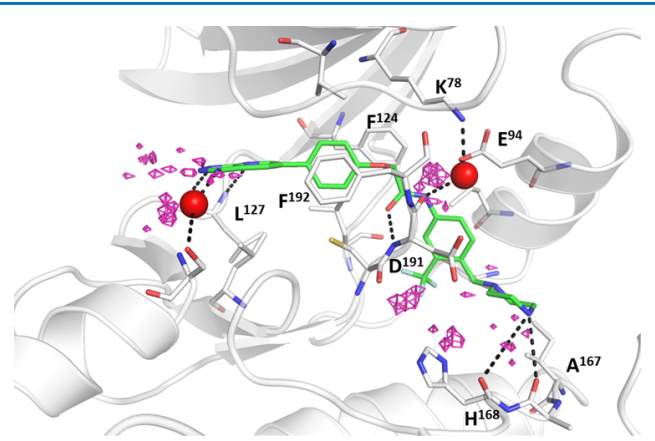

Figure 7. $\mathrm{MD}$ snapshots illustrating the interactions between compound 18 with Mnk1; active site residues of Mnk1/2 are shown as lines; hydrogen bonding interactions are indicated by the dashed lines (magenta). The kinase residues involved in hydrogen bond interactions are labeled accordingly. Water molecules involved in the protein-inhibitor interaction are shown as sphere (red), and the hydration sites are shown as a mesh (magenta).

hydration site corresponds to the linker binding region, where we found one, two, or three water molecules with high residence times. The water molecules interact with the amide linkers from the inhibitors and the sidechains of catalytic residues Lys78 (Mnk1)/Lys113 (Mnk2) and Glu94 (Mnk1)/ Glu129 (Mnk2) from the $\alpha \mathrm{C}$ helix. This highly ordered watermediated interaction was preserved in $\sim 95 \%$ of the simulation time (Figure 7).

Interestingly, the water molecules that we observed in the simulations of Mnk1/2-inhibitor complexes are conserved in many structures of active kinases bound to nucleotides and inhibitors and are involved in interactions between the catalytic residues Lys and Glu of the kinases with the nucleotide or inhibitors. We speculate that this water-mediated hydrogen bond network may contribute to the structural stability of the bound forms of the inhibitors.

Binding Free Energies. Binding free energies $(\Delta H)$ calculated between the 42 complexes of $M n k 1 / 2$ and inhibitors showed very poor correlation $\left(r^{2}=0.24\right.$ and 0.3 for Mnk1 and Mnk2, respectively) with $\mathrm{IC}_{50}$, although there was distinct improvement over the correlations from the docking scores. The inclusion of the entropy term $(T \Delta S)$ improved the correlation (Figure $5 \mathrm{c}, \mathrm{d})\left(r^{2}=0.47\right.$ and 0.46 for Mnkl and Mnk2, respectively); however, no linear relationship was observed (Table 2).

In the 42 complexes, it is clear that the nonbonded energies (van der Waals, electrostatics) and nonpolar solvation drive inhibitor binding to the Mnk1/2 kinases and are offset by unfavorable polar solvation and entropy. The nonpolar energies ( $\mathrm{vdW}+\mathrm{SA})$ contribute the most to binding ( $\sim 76$ to -55 $\mathrm{kcal} / \mathrm{mol}$ ) and appear to be correlated best with activity. This indicates that shape complementarity between the inhibitors and the binding pocket is the major determinant of binding. This term also separates the actives (contribution $\approx-81.7$ $\mathrm{kcal} / \mathrm{mol}$ ) from the inactives (contribution $\approx-65.3 \mathrm{kcal} / \mathrm{mol}$ ). Unfavorable contributions from the polar energies $\left(\Delta G_{\text {elec }}\right)$ suggest that desolvation opposes binding. An unfavorable entropy term $(-T \Delta S)$ contributes significantly, and the magnitude is similar to the electrostatic contributions. This is 
Table 2. Binding Free Energies of the Mnk1-Inhibitor Complexes Calculated Using the MMPBSA Method ${ }^{a, c, d, e}$

\begin{tabular}{|c|c|c|c|c|c|c|c|}
\hline compd & $\Delta E_{\text {elec }}$ & $\Delta E_{\mathrm{vdw}}$ & $\Delta G_{\mathrm{PB}}$ & $\Delta G_{\mathrm{SA}}^{b}$ & $\Delta G_{\text {pred(}(\mathrm{PB})}$ & $T \Delta S$ & $\mathrm{pIC}_{50} f$ \\
\hline 1 & -32.25 & -71.48 & 58.48 & -7.64 & -52.89 & -20.79 & 7.06 \\
\hline 2 & -21.83 & -68.77 & 53.82 & -7.29 & -44.06 & -19.34 & 7.05 \\
\hline 3 & -13.49 & -73.45 & 58.39 & -7.76 & -36.31 & -22.62 & 5.38 \\
\hline 4 & -23.61 & -80.11 & 72.69 & -7.63 & -38.67 & -22.40 & 5 \\
\hline 5 & -26.31 & -69.96 & 49.53 & -7.58 & -54.31 & -30.19 & 5 \\
\hline 6 & -19.24 & -73.44 & 54.16 & -7.70 & -46.23 & -25.99 & 5 \\
\hline 8 & -22.17 & -66.44 & 53.71 & -7.14 & -42.04 & -15.14 & 7.08 \\
\hline 9 & -12.64 & -64.90 & 46.03 & -7.58 & -39.08 & -25.33 & 5 \\
\hline 10 & -30.92 & -75.06 & 62.33 & -7.29 & -50.94 & -16.05 & 7.11 \\
\hline 11 & -23.10 & -71.51 & 59.68 & -7.36 & -42.29 & -25.61 & 7.23 \\
\hline 12 & -20.18 & -69.96 & 51.54 & -7.33 & -45.92 & -20.88 & 8.15 \\
\hline 13 & -41.34 & -76.92 & 60.06 & -7.33 & -65.53 & -22.25 & 7.21 \\
\hline 14 & -29.63 & -72.40 & 60.39 & -7.23 & -48.86 & -23.51 & 7.26 \\
\hline 16 & -39.55 & -76.71 & 64.48 & -7.37 & -59.14 & -21.92 & 7.32 \\
\hline 17 & -25.48 & -74.61 & 55.80 & -7.76 & -52.05 & -28.95 & 7.03 \\
\hline 18 & -33.21 & -77.06 & 65.50 & -7.56 & -52.33 & -24.33 & 5 \\
\hline 19 & -46.94 & -71.87 & 62.18 & -7.11 & -63.74 & -16.70 & 7.38 \\
\hline 20 & -13.08 & -62.27 & 41.98 & -7.36 & -40.72 & -24.06 & 5 \\
\hline 21 & -40.58 & -76.56 & 82.33 & -7.86 & -42.67 & -22.79 & 5 \\
\hline
\end{tabular}

${ }^{a}$ Mean energies are in kcal $/ \mathrm{mol}^{b}{ }^{b} \Delta G_{\mathrm{SA}}=\gamma \times \mathrm{SASA}+\beta ; \gamma=0.00542 \mathrm{kacl} / \mathrm{mol} \mathrm{A}{ }^{-2} ; \beta=0.92 \mathrm{kcal} / \mathrm{mol} .{ }^{c} \Delta H_{(\mathrm{PB})}=\Delta E_{\text {elec }}+\Delta E_{\mathrm{vdW}}+\Delta G_{\mathrm{SA}}+\Delta G_{\mathrm{PB}}$. ${ }^{d} \mathrm{~T} \Delta S=$ entropy changes. ${ }^{e} \Delta G_{\mathrm{pred}(\mathrm{PB})}($ calculated binding free energy by MMPBSA method $)=\Delta H_{(\mathrm{PB})}-T \Delta S .{ }_{\mathrm{pIC}} \mathrm{C}_{50}=\left(-\log \mathrm{IC}_{50}\right)$.

Table 3. Binding Free Energies of the Mnk2-Inhibitor Complexes Calculated Using the MMPBSA Method ${ }^{a, c, d, e}$

\begin{tabular}{|c|c|c|c|c|c|c|c|}
\hline compd & $\Delta E_{\text {elec }}$ & $\Delta E_{\mathrm{vdw}}$ & $\Delta G_{\mathrm{PB}}$ & $\Delta G_{\mathrm{SA}}^{b}$ & $\Delta G_{\text {pred }}$ & $T \Delta S$ & $\mathrm{pIC}_{50}{ }^{f}$ \\
\hline 1 & -17.72 & -73.85 & 65.67 & -8.08 & -33.98 & -20.40 & 7.88 \\
\hline 2 & -25.83 & -67.93 & 58.87 & -7.47 & -42.37 & -16.95 & 8.15 \\
\hline 3 & -30.52 & -69.88 & 64.11 & -7.69 & -43.98 & -25.02 & 5.5 \\
\hline 4 & -19.39 & -74.20 & 64.19 & -12.65 & -42.05 & -24.19 & 5.65 \\
\hline 5 & -21.11 & -69.51 & 56.81 & -7.70 & -41.51 & -17.26 & 5.22 \\
\hline 6 & -15.71 & -73.37 & 53.29 & -7.49 & -43.28 & -17.51 & 5.43 \\
\hline 8 & -28.59 & -71.26 & 64.53 & -7.28 & -42.59 & -19.78 & 8.22 \\
\hline 9 & -15.45 & -74.09 & 51.55 & -7.44 & -45.43 & -20.34 & 5.72 \\
\hline 10 & -22.92 & -65.52 & 52.59 & -7.42 & -43.28 & -15.06 & 8.09 \\
\hline 11 & -19.01 & -70.32 & 57.78 & -7.27 & -38.81 & -14.07 & 8.22 \\
\hline 12 & -34.48 & -73.48 & 64.72 & -7.31 & -50.55 & -13.32 & 8.22 \\
\hline 13 & -22.40 & -75.44 & 53.77 & -7.36 & -51.43 & -20.47 & 8.22 \\
\hline 14 & -31.64 & -69.38 & 60.26 & -7.04 & -47.81 & -16.06 & 8.15 \\
\hline 16 & -27.37 & -71.70 & 56.39 & -7.40 & -50.08 & -24.47 & 8.15 \\
\hline 17 & -38.90 & -77.08 & 66.75 & -7.66 & -56.89 & -14.35 & 8 \\
\hline 18 & -32.53 & -72.58 & 58.47 & -7.62 & -54.26 & -30.06 & 5.26 \\
\hline 19 & -26.88 & -75.27 & 59.45 & -7.23 & -49.93 & -17.10 & 8.22 \\
\hline 20 & -19.08 & -70.47 & 53.97 & -7.82 & -43.40 & -22.60 & 5.02 \\
\hline 21 & -20.28 & -75.59 & 65.68 & -8.04 & -38.22 & -26.90 & 5.86 \\
\hline
\end{tabular}

${ }^{a}$ Mean energies are in kcal $/ \mathrm{mol} .{ }^{b} \Delta G_{\mathrm{SA}}=\gamma \times \mathrm{SASA}+\beta ; \gamma=0.00542 \mathrm{kacl} / \mathrm{mol} \mathrm{A}{ }^{-2} ; \beta=0.92 \mathrm{kcal} / \mathrm{mol} .{ }^{c} \Delta H_{(\mathrm{PB})}=\Delta E_{\text {elec }}+\Delta E_{\mathrm{vdW}}+\Delta G_{\mathrm{SA}}+\Delta G_{\mathrm{PB}}$. $d_{T \Delta S}=$ entropy changes. ${ }^{e} \Delta G_{\text {pred(PB) }}$ (calculated binding free energy by MMPBSA method $)=\Delta H_{(\mathrm{PB})}-T \Delta S .{ }_{\mathrm{pIC}}^{f}=\left(-\log \mathrm{IC}_{50}\right)$.

expected because the ligands will have greater flexibility in the solution (Table 3).

Most residues from the binding pockets of the kinases make similar energetic contributions to binding in all complexes studied (Figure 8), further supporting the hypothesis used in our models, that all inhibitors adopt similar binding modes. Unsurprisingly, most favorable interactions are formed by residues in the active sites and in the allosteric sites of the kinases. These are either hydrophobic interactions or residues interacting with the inhibitor through hydrogen bonds. The above observations were also mirrored in the interactions of the Type I inhibitors bound to the active sites of the Mnk1/2 kinases, as reported earlier. ${ }^{15}$

Effect of Inclusion of Explicit Water Molecules during MMPBSA. Water can act both as a hydrogen bond donor and as an acceptor. Although the effects of water at biomolecular interfaces have been hard to quantify and have traditionally been ignored in $\mathrm{MM}(\mathrm{PB} / \mathrm{GB}) \mathrm{SA}$ calculations, recent studies 


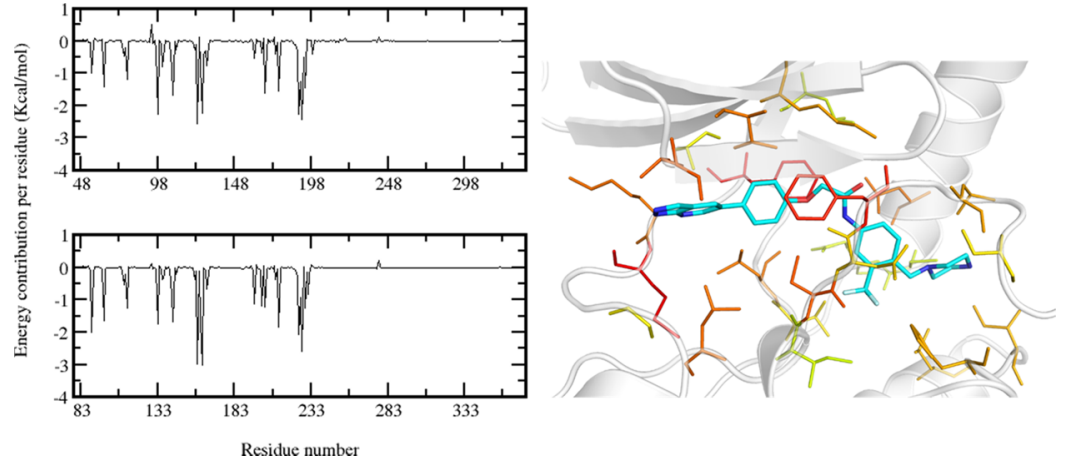

Figure 8. Per-residue decompositions of the binding free energies of compound 12 with Mnk1 (top left) and Mnk2 (bottom left). (Right) Structural mapping of contributions from residues to the binding. Only residues in the binding site are shown as lines and colored according to the contributions from red to blue corresponding to energetic contributions of -3.0 to $+3.0 \mathrm{kcal} / \mathrm{mol}$. Details of residue decomposition contribution is listed in Table 4.

treating water molecules as part of the receptor during molecular mechanics Poisson-Boltzmann surface area (MMPBSA) calculations have shown improved correlations between the predicted and experimental affinities. ${ }^{27-32}$ The problem gets more complex when there are multiple waters at the interfaces. Recently, Maffucci et al. ${ }^{27}$ have systematically investigated this by computing the MMGBSA affinities when different numbers of water molecules were explicitly included (10-50 water molecules) and found that in their system, 30 interfacial water molecules resulted in improved correlation between the predicted and experimental affinities by up to $30 \%$, compared to the calculations without waters.

The MM/PBSA calculations with the inclusion of explicit water molecules showed improved correlation with experimental $\mathrm{IC}_{50}$, with the correlation coefficient $\left(r^{2}\right)$ reaching values of 0.6 and 0.8 for Mnk1 and Mnk2, respectively (Figure 9). Although increased correlation was observed until about 10

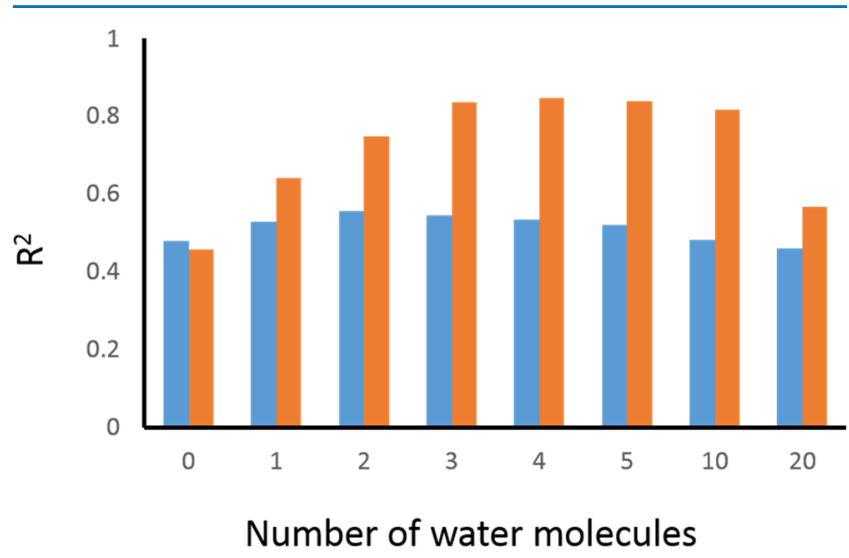

Figure 9. Correlation coefficient between experimental $\Delta G_{\text {bind }}$ and predicted binding energies obtained by MMPBSA analysis using different numbers of explicit water molecules for Mnk1 (blue) and Mnk2 (orange).

water molecules, it decreased upon the inclusion of 20 water molecules; similar observations have also been reported in other studies. ${ }^{27}$ Earlier studies have shown that the inclusion of 20-30 interfacial water molecules explicitly is optimal to yield the highest correlation between the MMPBSA and experimental binding affinities. The optimal number of explicit water molecules found here is less than what has been reported for other ligand-receptor complexes and is probably due to the smaller interface between the Mnk1/2 kinases and the Type II inhibitors(Table 4).

Table 4. Per-Residue Decomposition Energies of Each Mnk1/2-Inhibitor Complex Calculated by MMPBSA

\begin{tabular}{cccc} 
Mnk1 residues & $\Delta G_{\text {bind }}(\mathrm{kcal} / \mathrm{mol})$ & Mnk2 residues & $\Delta G_{\text {bind }}(\mathrm{kcal} / \mathrm{mol})$ \\
LEU54 & -0.993 & LEU89 & -1.995 \\
VAL62 & -1.421 & VAL97 & -1.647 \\
LYS77 & -1.183 & ALA110 & -0.534 \\
LEU97 & -2.283 & LYS112 & -1.261 \\
CYS100 & -0.805 & LEU132 & -1.748 \\
ILE106 & -0.837 & VAL141 & -0.833 \\
LEU107 & -1.692 & LEU142 & -1.684 \\
PHE123 & -2.557 & PHE158 & -2.98 \\
LYS125 & -0.806 & LYS160 & -1.701 \\
LEU126 & -2.238 & MET161 & -3.027 \\
GLY129 & -0.781 & GLY164 & -0.644 \\
LEU160 & -0.533 & LEU195 & -1.13 \\
HIE167 & -1.611 & ILE200 & -1.189 \\
LEU176 & -1.543 & HIE202 & -1.211 \\
CYS189 & -2.256 & LEU211 & -1.821 \\
ASP190 & -1.251 & CYS224 & -2.058 \\
PHE191 & -2.425 & ASP225 & -0.932 \\
LEU193 & -1.451 & PHE226 & -2.584 \\
& & LEU228 & -1.249 \\
& & SER230 & -0.822 \\
\hline
\end{tabular}

The active sites of Mnk1 and Mnk2 differ in 8 amino acids (as shown in Figure 3). In Mnk1, residues Leu54, Thr97, and Leu127 have branched sidechains without much conformational degrees of freedom and hence pack against the inhibitors. By contrast, Mnk2 has Val, Met and Met at the corresponding positions. These larger and linear sidechains in Mnk2 allow for flexibility which in turn appears to enable, in general, better packing of the inhibitors, by accommodating more water molecules; this number, of course, varies between compounds (Figure 9).

\section{CONCLUSIONS}

In this study, the interactions between the kinase domains of the Mnk1/2 kinases and a set of Type II inhibitors which bind to the inactive forms of the kinases are detailed using molecular modeling and MD simulations. Structural models of the catalytic domains of inactive (DFD-out) $M n k 1 / 2$ were built 
by homology and refined using MD simulations. An ensemble of simulated conformations was used to dock the Type II inhibitors into their binding sites. All compounds occupy the ATP binding site and an allosteric pocket with very similar binding modes. These complexes remained stable during MD simulations. Stability was governed by hydrophobic interactions between the inhibitors and the kinases and hydrogen bonds between the inhibitors and the catalytic Lys78, Asp191 (Mnk1), and Lys113, Asp226 (Mnk2) and Leu127 (Mnk1), Met162 (Mnk2) and Ala167 (Mnk1), Ala202 (Mnk2) modulating the stability. The hydrogen bonds appear to enhance the electrostatic interactions of the inhibitors, resulting in their improved binding affinities. Two distinct hydration sites that appear to further stabilize the ligand binding/interactions were observed. A decent correlation between the computed interaction energies and experimental affinities was found to be improved upon the inclusion of explicit water molecules. Binding of the inhibitors is largely driven by van der Waals packing against the hydrophobic residues in the binding sites and the associated nonpolar solvation. In summary, this study provides structural and energetic details of the interactions between a set of Type II inhibitors and inactive Mnk1/2 kinases in their DFD-out states, offering a basis for the design of new potent inhibitors with the novel finding that structural water molecules play an important role and should be considered when designing new inhibitors.

\section{MATERIALS AND METHODS}

The methodology adopted was as follows: (a) building the homology models of the Mnk1/2 kinases in their inactive conformations (given that there are no crystal structures); (b) MD simulations to refine the homology models; (c) preparation of and docking the Type II inhibitors into the active sites of an ensemble of the refined homology models of the Mnk1/2 kinases; (d) MD simulations of the complexes; (e) binding energy calculations; and (f) hydration analysis. The protocols used for sections $(\mathrm{a}-\mathrm{e})$ were the same as outlined in our earlier work on the binding of the Type I inhibitors to the catalytic domains of the Mnk1/2 kinases ${ }^{15}$ except that the template used for the homology models in the present study was the crystal structure of the inactive form of the Calmodulindomain protein kinase 1 (PDB code $2 \mathrm{WEI}) .{ }^{19} \mathrm{We}$ additionally used the structures of Mnk1 in its autoinhibited states (PDB ID id 2HW6), ${ }^{13}$ excluding the activation loop (because it occludes the binding pocket) and the structure of a mutant Mnk2 kinase which has been crystallized in the inactive form (PDB id $2 \mathrm{AC} 3)^{14}$ but is missing the activation loop; details of the methods are in the Supporting Information. We now outline the analysis of the roles of water molecules.

Selection of Interface Water Molecules. We investigated the effects of water molecules that stabilize the interactions between the kinases and the inhibitors. Each conformation sampled during the MD simulation was mined to identify water molecules (oxygens) that were located within $3.5 \AA$ of a kinase or inhibitor atom (nonhydrogen). These water molecules were considered to be part of the kinase structure during subsequent calculations of the binding free energies, using the MMPBSA method. ${ }^{33-35}$ We also investigated the effects of varying the numbers of water molecules included in each conformation sampled $(1,2,3,4,5,10$, and 20 waters).

\section{ASSOCIATED CONTENT}

\section{Supporting Information}

The Supporting Information is available free of charge on the ACS Publications website at DOI: 10.1021/acsomega.7b01403.

Crystal structures of Mnk1 and Mnk2 kinases; crystal structure of Mnk1 kinase in its autoinhibited form; predicted binding mode of compound 11 to Mnk1 in its active conformation; and details of the methods (PDF)

\section{AUTHOR INFORMATION}

\section{Corresponding Authors}

*E-mail: raghavk@bii.a-star.edu.sg. Phone: +65 6478 8353. Fax: +65 64789048 (S.K.).

*E-mail: chandra@bii.a-star.edu.sg. Phone: +65 64788273. Fax: +65 64789048 (C.S.V.).

ORCID

Srinivasaraghavan Kannan: 0000-0002-9539-5249

Joseph Cherian: 0000-0003-2663-7192

\section{Funding}

This work was financially supported by Biomedical Sciences Institutes (BMSI) and Joint Council Office (JCO project 1103 FG 07 05), Agency for Science, Technology and Research (A*STAR), Singapore, which is gratefully acknowledged.

\section{Notes}

The authors declare no competing financial interest.

\section{ACKNOWLEDGMENTS}

The authors thank A*STAR Computing Resource Centre $\left(A^{*} \mathrm{CRC}\right)$ for computing facilities.

\section{REFERENCES}

(1) Waskiewicz, A. J.; Johnson, J. C.; Penn, B.; Mahalingam, M.; Kimball, S. R.; Cooper, J. A. Phosphorylation of the cap-binding protein eukaryotic translation initiation factor $4 \mathrm{E}$ by protein kinase Mnk1 in vivo. Mol. Cell. Biol. 1999, 19, 1871-1880.

(2) Pyronnet, S. Phosphorylation of the cap-binding protein eIF4E by the MAPK-activated protein kinase Mnk1. Biochem. Pharmacol. 2000, 60, 1237-1243.

(3) Knauf, U.; Tschopp, C.; Gram, H. Negative regulation of protein translation by mitogen-activated protein kinase-interacting kinases 1 and 2. Mol. Cell. Biol. 2001, 21, 5500-5511.

(4) Hou, J.; Kam, F.; Proud, C. G.; Wang, S. Targeting Mnks for Cancer Therapy. Oncotarget 2012, 2, 118-131.

(5) Lim, S.; Saw, T. Y.; Zhang, M.; Janes, M. R.; Nacro, K.; Hill, J.; Lim, A. Q.; Chang, C.-T.; Fruman, D. A.; Rizzieri, D. A.; Tan, S. Y.; Fan, H.; Chuah, C. T. H.; Ong, S. T. Targeting of the MNK-eIF4E axis in blast crisis chronic myeloid leukemia inhibits leukemia stem cell function. Proc. Natl. Acad. Sci. U.S.A. 2013, 110, E2298-E2307.

(6) Hay, N. Mnk earmarks eIF4E for cancer therapy. Proc. Natl. Acad. Sci. U.S.A. 2010, 107, 13975-13976.

(7) Landon, A. L.; Muniandy, P. A.; Shetty, A. C.; Lehrmann, E.; Volpon, L.; Houng, S.; Zhang, Y.; Dai, B.; Peroutka, R.; MazanMamczarz, K.; Steinhardt, J.; Mahurkar, A.; Becker, K. G.; Borden, K. L.; Gartenhaus, R. B. MNKs act as a regulatory switch for eIF4E1 and eIF4E3 driven mRNA translation in DLBCL. Nat. Commun. 2014, 5, 5413.

(8) Teo, T.; Yu, M.; Yang, Y.; Gillam, T.; Lam, F.; Sykes, M. J.; Wang, S. Pharmacologic Co-Inhibition of Mnks and mTORC1 Synergistically Suppresses Proliferation and Perturbs Cell Cycle Progression in Blast Crisis-Chronic Myeloid Leukemia Cells. Cancer Lett. 2015, 357, 612-623.

(9) Lock, R.; Ingraham, R.; Maertens, O.; Miller, A. L.; Weledji, N.; Legius, E.; Konicek, B. M.; Yan, S.-C. B.; Graff, J. R.; Cichowski, K. 
Cotargeting MNK and MEK Kinases Induces the Regression of NF1Mutant Cancers. J. Clin. Invest. 2016, 126, 2181-2190.

(10) Bianchini, A.; Loiarro, M.; Bielli, P.; Busà, R.; Paronetto, M. P.; Loreni, F.; Geremia, R; Sette, C. Phosphorylation of eIF4E by MNKs Supports Protein Synthesis, Cell Cycle Progression and Proliferation in Prostate Cancer Cells. Carcinogenesis 2008, 29, 2279-2288.

(11) Webster, K. R.; Goel, V. K.; Hung, I. N. J.; Parker, G. S.; Staunton, J.; Neal, M.; Molter, J.; Chiang, G. G.; Jessen, K. A.; Wegerski, C. J.; Sperry, S.; Huang, V.; Chen, J.; Thompson, P. A.; Appleman, J. R.; Webber, S. E.; Sprengeler, P. A.; Reich, S. H. eFT508, a potent and selective mitogen-activated protein kinase interacting kinase (MNK) 1 and 2 inhibitor, is efficacious in preclinical models of diffuse large B-cell lymphoma (DLBCL). 57th American Society of Hematology Annual Meeting and Exposition: Orlando, FL, 2015, Session 625 .

(12) Klar, U.; Wortmann, L.; Kettschau, G.; Graham, K.; Richter, A.; Lienau, P.; Puehler, F.; Petersen, K.; Siegel, F.; Sülzle, D. Thienopyrimidines as mknk1 and mknk2 Inhibitors. WO2015/ 074986A1, 2015.

(13) Jauch, R.; Cho, M.-K.; Jäkel, S.; Netter, C.; Schreiter, K.; Aicher, B.; Zweckstetter, M.; Jäckel, H.; Wahl, M. C. Mitogen-activated protein kinases interacting kinases are autoinhibited by a reprogrammed activation segment. EMBO J. 2006, 25, 4020-4032.

(14) Jauch, R.; Jäkel, S.; Netter, C.; Schreiter, K.; Aicher, B.; Jäckle, H.; Wahl, M. C. Crystal structures of the Mnk2 kinase domain reveal an inhibitory conformation and a zinc binding site. Structure 2005, 13, $1559-1568$

(15) Kannan, S.; Poulsen, A.; Yang, H. Y.; Ho, M.; Ang, S. H.; Eldwin, T. S. W.; Jeyaraj, D. A.; Chennamaneni, L. R.; Liu, B.; Hill, J.; Verma, C. S.; Nacro, K. Probing the Binding Mechanism of Mnk Inhibitors by Docking and Molecular Dynamics Simulations. Biochemistry 2015, 54, 32-46.

(16) Cherian, J.; Nacro, K.; Poh, Z. Y.; Guo, S.; Jeyaraj, D. A.; Wong, Y. X.; Ho, M.; Yang, H. Y.; Joy, J. K.; Kwek, Z. P.; Liu, B.; Wee, J. L. K.; Ong, E. H.; Choong, M. L.; Poulsen, A.; Lee, M. A.; Pendharkar, V.; Ding, L. J.; Manoharan, V.; Chew, Y. S.; Sangthongpitag, K.; Lim, S.; Ong, S. T.; Hill, J.; Keller, T. H. Structure-Activity Relationship Studies of Mitogen Activated Protein Kinase Interacting Kinase (MNK) 1 and 2 and BCR-ABL1 Inhibitors Targeting Chronic Myeloid Leukemic Cells. J. Med. Chem. 2016, 59, 3063-3078.

(17) Nacro, K.; Chennamaneni, L. R.; Cherian, J.; Poulsen, A. Heteroaryl Alkyne Derivatives and Uses Thereof. WO2015/ 108490A2, 2015.

(18) Cherian, J.; Duraiswamy, A. J.; Nacro, K. Compounds Including Map Kinase Interacting Kinases 1 and 2 (mnk1 and mnk2) Modulators and $\mathrm{Abl}$ and $\mathrm{Abl}(\mathrm{t} 315 \mathrm{i})$ Inhibitors, and Uses Thereof. WO2014/088519A1, 2014.

(19) Artz, J. D.; Wernimont, A. K.; Allali-Hassani, A.; Zhao, Y.; Amani, M.; Lin, Y.-H.; Senisterra, G.; Wasney, G. A.; Fedorov, O.; King, O.; Roos, A.; Lunin, V. V.; Qiu, W.; Finerty, P., Jr.; Hutchinson, A.; Chau, I.; von Delft, F.; MacKenzie, F.; Lew, J.; Kozieradzki, I.; Vedadi, M.; Schapira, M.; Zhang, C.; Shokat, K.; Heightman, T.; Hui, R. The Cryptosporidium parvum kinome. BMC Genomics 2011, 12, 478.

(20) Woods, C. J.; Malaisree, M.; Long, B.; McIntosh-Smith, S.; Mulholland, A. J. Computational assay of H7N9 influenza neuraminidase reveals $\mathrm{R} 292 \mathrm{~K}$ mutation reduces drug binding affinity. Sci. Rep. 2013, 3, 3561.

(21) Xing, L.; Klug-Mcleod, J.; Rai, B.; Lunney, E. A. Kinase hinge binding scaffolds and their hydrogen bond patterns. Bioorg. Med. Chem. 2015, 23, 6520-6527.

(22) Hu, J.; Ahuja, L. G.; Meharena, H. S.; Kannan, N.; Kornev, A. P.; Taylor, S. S.; Shaw, A. S. Kinase regulation by hydrophobic spine assembly in cancer. Mol. Cell. Biol. 2015, 35, 264-276.

(23) Barillari, C.; Duncan, A. L.; Westwood, I. M.; Blagg, J.; van Montfort, R. L. Analysis of water patterns in protein kinase binding sites. Proteins: Struct., Funct., Bioinf. 2011, 79, 2109-2121.
(24) Robinson, D. D.; Sherman, W.; Farid, R. Understanding kinase selectivity through energetic analysis of binding site waters. ChemMedChem 2010, 5, 618-627.

(25) Levinson, N. M.; Boxer, S. G. A conserved water-mediated hydrogen bond network defines bosutinib's kinase selectivity. Nat. Chem. Biol. 2014, 10, 127-132.

(26) Fernández, A.; Sanguino, A.; Peng, Z.; Ozturk, E.; Chen, J.; Crespo, A.; Wulf, S.; Shavrin, A.; Qin, C.; Ma, J.; Trent, J.; Lin, Y.; Han, H.-D.; Mangala, L. S.; Bankson, J. A.; Gelovani, J.; Samarel, A.; Bornmann, W.; Sood, A. K.; Lopez-Berestein, G. An anticancer C-Kit kinase inhibitor is reengineered to make it more active and less cardiotoxic. J. Clin. Invest. 2007, 117, 4044-4054.

(27) Maffucci, I.; Contini, A. Explicit Ligand Hydration Shells Improve the Correlation between MM-PB/GBSA Binding Energies and Experimental Activities. J. Chem. Theory Comput. 2013, 9, 27062717.

(28) Zhu, Y.-L.; Beroza, P.; Artis, D. R. Including Explicit Water Molecules as Part of the Protein Structure in MM/PBSA Calculations. J. Chem. Inf. Model. 2014, 54, 462-469.

(29) Mikulskis, P.; Genheden, S.; Ryde, U. Effect of Explicit Water Molecules on Ligand-Binding Affinities Calculated with the MM/ GBSA Approach. J. Mol. Model. 2014, 20, 2273.

(30) Genheden, S.; Ryde, U. The MM/PBSA and MM/GBSA Methods to Estimate Ligand-Binding Affinities. Expert Opin. Drug Discovery 2015, 10, 449-461.

(31) Wong, S.; Amaro, R. E.; McCammon, J. A. MM-PBSA Captures Key Role of Intercalating Water Molecules at a Protein-Protein Interface. J. Chem. Theory Comput. 2009, 5, 422-429.

(32) Hou, T. J.; Wang, J.; Li, Y.; Wang, W. Assessing the performance of the molecular mechanics/Poisson Boltzmann surface area and molecular mechanics/generalized Born surface area methods. II. The accuracy of ranking poses generated from docking. J. Comput. Chem. 2011, 32, 866-877.

(33) Homeyer, N.; Gohlke, H. Free Energy Calculations by the Molecular Mechanics Poisson-Boltzmann Surface Area Method. Mol. Inf. 2012, 31, 114-122.

(34) Wang, J.; Hou, T.; Xu, X. Recent advances in free energy calculations with a combination of molecular mechanics and continuum models. Curr. Comput.-Aided Drug Des. 2006, 2, 287-306.

(35) Hou, T.; Wang, J.; Li, Y.; Wang, W. Assessing the performance of the molecular mechanics/Poisson Boltzmann surface area and molecular mechanics/generalized Born surface area methods. II. The accuracy of ranking poses generated from docking. J. Comput. Chem. 2011, 32, 866-877. 